\title{
Quantum creation of an Inhomogeneous universe
}

\author{
Sigbjørn Hervik* \\ Department of Physics, University of Oslo \\ P.O.Box 1048 Blindern \\ N-0316 Oslo, Norway
}

\begin{abstract}
In this paper we study a class of inhomogeneous cosmological models which is a modified version of what is usually called the Lemaitre-Tolman model. We assume that we have a space with 2-dimensional locally homogeneous spacelike surfaces. In addition we assume they are compact. Classically we investigate both homogeneous and inhomogeneous spacetimes which this model describe. For instance one is a quotient of the $\mathrm{AdS}_{4}$ space which resembles the BTZ black hole in $\mathrm{AdS}_{3}$.

Due to the complexity of the model we indicate a simpler model which can be quantized easily. This model still has the feature that it is in general inhomogeneous. How this model could describe a spontaneous creation of a universe through a tunneling event is emphasized.
\end{abstract}

\section{INTRODUCTION}

In the eighties several authors [1] 6] suggested that the universe could have been spontaneously created though a tunneling event. What the nature of such a tunneling event could be is still unsettled. Today's theories suggest however that shortly after the Planck era the universe was in an arbitrary state. A homogeneous and isotropic universe is believed to be a rather special configuration, so an arbitrary state suggests the universe was inhomogeneous. Recently the question of topology 7 has been added into the discussion. What is the most probable topology of the universe? We know that there exist a lot more hyperbolic manifolds than elliptic and flat ones [8]. Do we therefore live in a hyperbolic universe?

In this paper we shall study a class of cosmological models which has 2-dimensional locally homogeneous compact spacelike surfaces. The 2-dimensional compact oriented surfaces can be classified in term of an integer $g$, the genus of the surface. There is one elliptic surface $g=0$, one flat $g=1$ and a infinite of hyperbolic surfaces $g \geq 2$.

\footnotetext{
*e-mail:sigbjorn.hervik@fys.uio.no

${ }^{1}$ See for instance [7].
} 
We write the metric in a similar form to that of Kuchař [9] and Kenmoku and collaborators (KKTY) [10]:

$$
d s^{2}=-N^{2} d t^{2}+Q^{2}\left(d r+N^{r} d t\right)^{2}+R^{2} d \Omega_{g}^{2}
$$

$N, N^{r}, Q$ and $R$ are all functions of $r$ and $t$, and $d \Omega_{g}^{2}$ is the metric the $g$-holed torus?, $T_{g}$. These metrics cover a great variety of different spatial topologies. In terms of the Thurston classification [11], their covering spaces are for instance: $E^{3}, S^{3}, \mathbb{H}^{3}, \mathbb{R} \times S^{2}$ and $\mathbb{R} \times \mathbb{H}^{2}$. Thus a great variety of cosmological models are incorporated in the metric (1). Our main concern will however be the Lemaitre-Tolman models (or LT for short). In the LT models $g=0$. This case includes the first three of the Thurston geometric models. The case where $R^{\prime}=Q^{\prime}=N^{\prime}=0$ are the Kantowski-Sachs $(g=0)$ metric and their hyperbolic versions $(g \geq 2)$ studied by Fugandes [12]. Throughout this paper we will denote all the cases $\mathbb{R} \times T_{g}$ as KS. This model will link the models studied in the paper by Nambu and Sasaki [13 with for instance the homogeneous FRW models or the Einstein deSitter models. This model can therefore among others describe a quantum tunneling of a universe from a Schwarzschild Black Hole into a deSitter universe.

We will choose the curvature of $T_{g}$ to be $\kappa=1,0,-1$ for $g=0, g=1$ and $g \geq 2$ respectively. The Gauss-Bonnet Theorem then implies

$$
\mathcal{A}_{g} \kappa=4 \pi(1-g)
$$

where $\mathcal{A}_{g}$ is the area of the $g$-holed torus $T_{g}$. If we choose $\mathcal{A}_{1}=4 \pi$ we can write

$$
\mathcal{A}_{g}=4 \pi n_{g}
$$

where

$$
n_{g}= \begin{cases}1, & g=0,1 \\ (g-1), & g \geq 2\end{cases}
$$

From the action of pure gravity $S_{G}=\frac{1}{16 \pi} \int d^{4} x(R-2 \Lambda)$, we obtain for these models

$$
\begin{gathered}
S_{G}=\int d t \int d r n_{g}\left[-N^{-1}\left(R\left(-\dot{Q}+\left(Q N^{r}\right)^{\prime}\right)\left(-\dot{R}+R^{\prime} N^{r}\right)+\frac{1}{2} Q\left(-\dot{R}+R^{\prime} N^{r}\right)^{2}\right)\right. \\
\left.+N\left(-Q^{-1} R R^{\prime \prime}-\frac{1}{2} Q^{-1} R^{\prime 2}+Q^{-2} R R^{\prime} Q^{\prime}+\frac{1}{2} Q\left(\kappa-\Lambda R^{2}\right)\right)\right]
\end{gathered}
$$

\footnotetext{
${ }^{2}$ Note a slightly different notation compared to the notation used by KKTY and Kuchař

${ }^{3}$ Or more correctly: $d \Omega_{g}^{2}$ is a metric on the $g$-holed torus, $T_{g}$. Since the Teichmüller space for $g \geq 1$ have dimension greater than 1 , there are an uncountable number of non-isometric metrics on $T_{g}, g \geq 1$.
}

${ }^{4}$ In this paper we have used the convention: $c=G_{N}=\hbar=k_{B}=1$. 
where the prime and dot denote the derivative with respect to $r$ and $t$ respectively. A cosmological constant is also included. We will also consider a matter term or source term of the form:

$$
S_{D}=-4 \pi n_{g} \int d t \int d r N \tilde{\rho}
$$

where $\tilde{\rho}$ an independent function. We introduce this source function because it is one of the simplest ways to generate inhomogeneities. In the presence of classical dust this function $\tilde{\rho}$ could be related to the dust density $\rho$ by the equation $\tilde{\rho}=Q R^{2} \rho$. Thus in this description $\tilde{\rho}$ is not a dynamical matter field, we have basically solved the matter equations and reinserted the solutions into the Lagrangian .

The total action is now assumed to be $S=S_{G}+S_{D}$. The canonical momenta are:

$$
\begin{aligned}
& P_{Q}=-n_{g} \frac{R}{N}\left(\dot{R}-R^{\prime} N^{r}\right) \\
& P_{R}=-n_{g} \frac{1}{N}\left[R\left(\dot{Q}-\left(Q N^{r}\right)^{\prime}\right)+Q\left(\dot{R}-R^{\prime} N^{r}\right)\right]
\end{aligned}
$$

Through a Legendre transform we can bring the action into a canonical form:

$$
S=\int d t \int d r\left[P_{R} \dot{R}+P_{Q} \dot{Q}-\left(N \mathfrak{H}+N^{r} \mathfrak{H}_{r}\right)\right]
$$

where

$$
\begin{aligned}
\mathfrak{H}= & -\frac{1}{n_{g} R} P_{Q} P_{R}+\frac{1}{2 n_{g}} \frac{Q}{R^{2}} P_{Q}^{2} \\
& +n_{g}\left[\frac{R R^{\prime \prime}}{Q}-\frac{R R^{\prime} Q^{\prime}}{Q^{2}}+\frac{1}{2} \frac{R^{\prime 2}}{Q}-\frac{1}{2} \kappa Q+\frac{1}{2} \Lambda Q R^{2}+4 \pi \tilde{\rho}\right] \\
\mathfrak{H}_{r}= & R^{\prime} P_{R}-Q P_{Q}^{\prime}
\end{aligned}
$$

\section{THE CONSTRAINTS IN THE KSLT MODEL}

From the canonical form of the action we can readily write down the energy constraint and the momentum constraint:

$$
\mathfrak{H}=0, \quad \mathfrak{H}_{r}=0
$$

It is also useful to study a special combination of these two constraints,

$$
-\frac{R^{\prime}}{Q} \mathfrak{H}-\frac{P_{Q}}{R Q} \mathfrak{H}_{r}=n_{g}\left(M^{\prime}-m^{\prime}\right)=0
$$

\footnotetext{
${ }^{5}$ If we want to treat dust as a dynamical field we get a term in the Lagrangian that is linear in the conjugated momentum of the dust field. See for instance [14, 15].
} 
where $M$ is called the mass function and is defined by

$$
M \equiv-\frac{1}{2}\left[-\frac{P_{Q}^{2}}{R n_{g}^{2}}+\frac{R R^{\prime 2}}{Q^{2}}-\kappa R+\frac{\Lambda}{3} R^{3}\right]
$$

and $m$ is defined by

$$
m \equiv \int^{r} d r 4 \pi \tilde{\rho} \frac{R^{\prime}}{Q}
$$

Inserting the relation $\tilde{\rho}=\rho R^{2} Q$ we see that in the case $\kappa=1$ we can interpret the function $m$ as the mass of the dust inside a spherical shell of radius $R$.

If we calculate the equal-time Poisson algebra we obtain:

$$
\begin{aligned}
\left\{\mathfrak{H}_{r}(r), \mathfrak{H}_{r}\left(r^{*}\right)\right\} & =\mathfrak{H}_{r}(r) \delta^{\prime}\left(r-r^{*}\right)-\mathfrak{H}_{r}\left(r^{*}\right) \delta^{\prime}\left(r^{*}-r\right) \\
\left\{\mathfrak{H}(r), \mathfrak{H}_{r}\left(r^{*}\right)\right\} & =\mathfrak{H}^{\prime}(r) \delta\left(r-r^{*}\right)+\mathfrak{H}^{\prime}(r) \delta^{\prime}\left(r-r^{*}\right) \\
\left\{\mathfrak{H}_{r}(r), \mathfrak{H}_{r}\left(r^{*}\right)\right\} & =\mathfrak{H}_{r}(r) \delta^{\prime}\left(r-r^{*}\right)-\mathfrak{H}_{r}\left(r^{*}\right) \delta^{\prime}\left(r^{*}-r\right) \\
\left\{\mathfrak{H}(r), \mathfrak{H}^{*}\left(r^{*}\right)\right\} & =Q(r)^{-2} \mathfrak{H}_{r}(r) \delta^{\prime}\left(r-r^{*}\right)-Q\left(r^{*}\right)^{-2} \mathfrak{H}_{r}\left(r^{*}\right) \delta^{\prime}\left(r^{*}-r\right) \\
\left\{M(r), \mathfrak{H}_{r}\left(r^{*}\right)\right\} & =M^{\prime}(r) \delta\left(r-r^{*}\right) \\
\left\{M(r), \mathfrak{H}\left(r^{*}\right)\right\} & =-Q(r)^{-3} R^{\prime}(r) \mathfrak{H}_{r}(r) \delta\left(r-r^{*}\right) \\
\left\{M(r), M\left(r^{*}\right)\right\} & =0
\end{aligned}
$$

Thus according to the above equations the mass function is a constant of motion in a weak sense.

\section{CLASSICAL SOLUTIONS}

For simplicity, let us work in the gauge $N^{r}=0$. But for the time being $N$ is still arbitrary. The constraint $\mathfrak{H}_{r}$ can easily be solved in quadrature. Using the expressions for the canonical momenta, eq. 7 and 8, combined with the constraint equation 10, and simplifying yields the differential equation:

$$
R^{\prime} \dot{Q}-Q \dot{R}^{\prime}+\dot{R} Q \frac{N^{\prime}}{N}=0
$$

which has the solution $R^{\prime}=F Q$. The function $F$ satisfies

$$
R^{\prime} \dot{F}-F \dot{R} \frac{N^{\prime}}{N}=0
$$

For the present time we have to be a bit careful when we solve this equation. If $R^{\prime}=0$ which happens in the KS case, the momentum constraint yields $N^{\prime}=0$. For $R^{\prime} \neq 0$ we can solve the equation for $F$ :

$$
F(r, t)=f(r) \exp \left[\int^{t}\left(\frac{\dot{R}}{R^{\prime}} \frac{N^{\prime}}{N}\right) d t\right]
$$


and $f(r)$ is an arbitrary function of $r$. We can add these results together and keep the above expression for $F(r, t)$ but with the additional restriction $f(r)=0$ whenever $R^{\prime}=0$. We will ignore the possibility that the term $\frac{N^{\prime}}{R^{\prime}}$ in the exponential function causing trouble if there exists a $t^{*}$ and a $r^{*}$ such that $R^{\prime}\left(t^{*}, r^{*}\right)=0$, then $F\left(t, r^{*}\right)=0$ for all $t$.

The mass function is always well defined and turns into:

$$
M=\frac{R \dot{R}^{2}}{2 N^{2}}+\frac{1}{2}\left(\kappa-F^{2}\right) R-\frac{\Lambda}{6} R^{3}=m
$$

where $m(r)=\int^{r} d r 4 \pi \tilde{\rho} F$. If $\dot{F}=0$ the mass equation (19) is separable and can be solved in quadrature.

\section{A. Time independent solutions}

Let us assume $\dot{R}=0$. This implies $\dot{F}=0$. Solving the mass equation with respect to $F$ yields:

$$
F^{2}=\kappa-\frac{2 m(r)}{R}-\frac{\Lambda}{3} R^{2}
$$

In order to find the lapse $N$ we have to use the equation for $\dot{P}_{R}$ :

$$
\begin{aligned}
-\frac{1}{N} \dot{P}_{Q} & =\frac{1}{N}\left\{P_{Q}, \int d r N \mathfrak{H}\right\} \\
& =\frac{1}{2 n_{g}} \frac{P_{Q}^{2}}{R^{2}}+n_{g}\left(\frac{1}{2} \frac{R^{\prime 2}}{Q^{2}}-\frac{1}{2}\left(\kappa-\Lambda R^{2}\right)+\frac{N^{\prime} R R^{\prime}}{N Q^{2}}\right)
\end{aligned}
$$

Inserting $F^{2}$ into the equation for $P_{Q}$ yields the following equation for the lapse $N$ :

$$
\frac{N^{\prime}}{N}=\frac{1}{2}\left(-\frac{1}{R}+\frac{\kappa-\Lambda R^{2}}{\kappa R-2 m-\frac{\Lambda}{3} R^{3}}\right) R^{\prime}
$$

As we see, we might as well (at least locally) consider $R$ as our new radial variable, i.e. $R=r$. If we do so, the solution to the above equation can be written as:

$$
N^{2}=\eta^{2}(t) \frac{1}{r} e^{I}
$$

where

$$
I=\int^{r} d r\left(\frac{\kappa-\Lambda r^{2}}{\kappa r-2 m(r)-\frac{\Lambda}{3} r^{3}}\right)
$$

and $\eta(t)$ is an arbitrary function of $t$. Thus

\footnotetext{
${ }^{6}$ We get a “递"-expression for $\frac{N^{\prime}}{R^{\prime}}$.
} 


$$
d s^{2}=-\eta^{2}(t) \frac{1}{r} e^{I} d t^{2}+\frac{d r^{2}}{\kappa-\frac{2 m(r)}{r}-\frac{\Lambda}{3} r^{2}}+r^{2} d \Omega_{g}^{2}
$$

wherever $\kappa-\frac{2 m(r)}{r}-\frac{\Lambda}{3} r^{2}>0$

If for instance we have constant dust density, i.e. $m(r)=\mu+\frac{4}{3} \pi \hat{\rho} r^{3}$ the integral may be evaluated in terms of elementary functions [16].

\section{Spatial topologies in $A d S_{4}$ space}

For all the deSitter spaces $(m=0)$ the integral $I$ is simply:

$$
I=\ln \left(\kappa r-\frac{\Lambda}{3} r^{3}\right)
$$

for physical spacetimes. We notice that $\kappa \leq 0$ is not compatible with a $\Lambda>0$. But for $\Lambda<0$ we may have any $\kappa$. All of these spaces will have spatial sections which have constant negative curvature, i.e. they belong to the Thurston classification $\mathbb{H}^{3}$. The cases $\kappa=0,-1$ are not compatible with compact spatial sections, but $\kappa=1$ may have compact spatial sections if we demand that the metric 23 is defined locally in $r$. If we demand that the metric should be globally defined then we obtain the space $\mathbb{H}^{3}$ itself. Unfortuneatly a classification of the possible compact quotients of $\mathbb{H}^{3}$ is still far from being established. Let us for simplicity's sake set $\Lambda=-3$.

- $\underline{\kappa=0:}$ This is the non-compact multiply connected space investigated by Sokoloff and Starobinskii [19]. This space is best illustrated in the Poincaré half space model 8 . If we do the coordinate transformation $r=-\frac{1}{z}$ the metric turns into

$$
d s^{2}=\frac{1}{z^{2}}\left(-d t^{2}+d x^{2}+d y^{2}+d z^{2}\right)
$$

which is manifest on Poincaré half space form? We identify points along two pairs of planes parallel to the $z$-axis. It is easily seen that this space does not allow compactification in the $z$ (or $r$ ) direction.

- $\underline{\kappa=-1}$ : This section of the hyperbolic space can be illustrated as follows. Draw two $4 g$-gons on the boundary of the Poincare ball model. These polygons can be placed so that they are symmetric to each other through the origin. Connecting the polygons using geodetic planes we obtain a space that look like an apple core(see figure 22). Also

\footnotetext{
${ }^{7}$ Note that in general $\hat{\rho} \neq \rho$. We define $\hat{\rho}$ so that it is not a funtion of $t$, while $\rho$ in general could be a function of $t$.

${ }^{8}$ See figure 1.

${ }^{9}$ Doing a Wick rotation of the time component, $\tau=i t$, we obtain the "Euclidean" section of $\mathrm{AdS}_{4}$ space. Here we see that the Euclidean section is the 4-dimensional hyperbolic space $\mathbb{H}^{4}$.
} 
this case will not allow a compactification in the $r$ direction. Actually we have cheated a little bit, because the space contains a horizon with topology as a $g$-torus, much like that of a black hole [17]. The area of this horizon is

$$
A_{g}=\frac{12 \pi}{|\Lambda|} n_{g}
$$

Thus apparently we can relate to it the Bekenstein-Hawking entropy:

$$
S_{B H}=\frac{1}{4} \int_{T_{g}} d A=\frac{3 \pi}{|\Lambda|} n_{g}
$$

This metric is very similar to the BTZ black hole in $\mathrm{AdS}_{3}$ [18]. Including a mass term in this case is straight forward. Note also that in the above case the "Black Hole" entropy is a feature of the negatively curved compact spaces $T_{g}$. To obtain the $\mathrm{AdS}_{4}$ apple we have actually taken two such spaces and glued them along their horizons to obtain a topological complete space. Note also that the horizon is the only $r=$ constant surface which is totally geodesic. To ensure regularity of the total space after we have performed a gluing we have to glue along totally geodesic surfaces.

If we had demanded that all of these cases should be simply connected, they would have yielded the same space $\mathbb{H}^{3}$. But since we have assumed that our locally homogeneous 2dimensional surfaces are compact all of the above cases yield topologically different spaces. Again [20] we see that assuming compact 2-dimensional locally homogeneous spaces ensure that the different cases will yield different solutions.

\section{B. Kantowski-Sachs-like solutions}

In the Kantowski-Sachs case we have $R^{\prime}=0$. This implies $F=0$. The mass equation will now yield:

$$
\mu=\frac{R \dot{R}^{2}}{2 N^{2}}+\frac{1}{2} \kappa R-\frac{\Lambda}{6} R^{3}
$$

where $\mu$ is a constant. Inserting this into the energy constraint we obtain an equation similar to the equation 22 :

$$
\frac{\dot{Q}}{Q}=\frac{1}{2}\left(-\frac{1}{R}+\frac{\Lambda R^{2}+8 \pi \rho R^{2}-\kappa}{\frac{\Lambda}{3} R^{3}+2 \mu-\kappa R}\right) \dot{R}
$$

Again we see that we can equally well consider $R$ to be $R=t$. This equation is very similar to the equation 22. This is not surprising since if we smoothly continue the Schwarzschild metric to the region inside the black hole horizon we obtain the KS metric inside the horizon 13. Smooth continuing the deSitter solution to the region outside the deSitter horizon gives the KS metric [21]. We also note that the $t \leftrightarrow r$ symmetry is manifestly broken with the inclusion of classical dust. Due to the conservation of the rest mass of the dust, we would obtain $\int_{\Sigma} d^{3} x \rho Q R^{2}=V_{c} \mathcal{M}$ where $V_{c}=\int_{\Sigma} d^{3} x$ is the coordinate volume if the spatial sections $\Sigma$ are compact. 
We re-write these equations for a general dust distribution. We assume that the topology in the $r$-direction is that of a circle $S^{1}$. If $0 \leq r \leq \xi$ then we can write

$$
\tilde{\rho}=\mathcal{M}+\sum_{n=1}^{\infty}\left[a_{n}(t) \cos \left(\frac{2 \pi n r}{\xi}\right)+a_{-n}(t) \sin \left(\frac{2 \pi n r}{\xi}\right)\right]
$$

We use the same trick to write $\left(\frac{\Lambda}{3} t^{2}+\frac{2 \mu}{t}-\kappa\right)^{-\frac{1}{2}} Q=G$ where

$$
G(r, t)=\sum_{n=0}^{\infty}\left[c_{n}(t) \cos \left(\frac{2 \pi n r}{\xi}\right)+c_{-n}(t) \sin \left(\frac{2 \pi n r}{\xi}\right)\right]
$$

Since the cosines and the sines form a complete and orthogonal set of functions on the circle the equations for $c_{n}, n \neq 0$ are

$$
\dot{c}_{n}(t)=\frac{4 \pi a_{n}(t)}{t\left(\frac{\Lambda}{3} t^{2}+\frac{2 \mu}{t}-\kappa\right)^{\frac{3}{2}}}
$$

The equation for $c_{0}(t)$ is

$$
\dot{c}_{0}(t)=\frac{4 \pi \mathcal{M}}{t\left(\frac{\Lambda}{3} t^{2}+\frac{2 \mu}{t}-\kappa\right)^{\frac{3}{2}}}
$$

can be integrated at once by elementary calculus.

In the absence of dust $\tilde{\rho}=0$ this reduces to the ordinary inner/outer solutions of the Schwarzschild-deSitter spacetime. This is just the homogeneous solution of the KS metric. If there are inhomogeneities we have to solve the equations for $c_{n}(t), n \neq 0$. Unfortunately we then have to know the evolution of the matter content. If we on the other hand knew the metric, i.e. the functions $c_{n}(t)$ we could uniquely determine the matter distribution.

\section{LT SOLUTIONS}

So far we have only investigated time-independent and KS-like solutions. We saw that they represented different sections of a more general spacetime. Let us now look at the LT

solutions, which we can obtain if we choose the universal time gauge $N=1$. The coordinates now follow the collapsing/expanding dust.

If we choose $N=1, F=F(r)$ will follow. We can now write the mass equation as an "energy" equation:

$$
\frac{1}{2} \dot{R}^{2}+V(r, R)=E(r)
$$

where the "potential" $V$ and the "energy" $E$ are given by

$$
\begin{aligned}
& V=-\frac{m}{R}-\frac{\Lambda}{6} R^{2} \\
& E=-\frac{1}{2}\left(\kappa-F^{2}\right)
\end{aligned}
$$


This energy equation may be integrated and solved exactly. A summary of the results and a qualitative description of the physical meaning of the solutions is given in 22]. The solutions may be written in terms of the Weierstrass' elliptic functions [23]. The actual expressions are not very informative unless the reader has massive knowledge of these elliptic functions. However a lot of qualitative information can be extracted from simple classical considerations.

We will however solve the energy equation in the big and small $R$ limit.

\section{Large $R$ : Neglecting the $m$ term}

If we neglect the $m$ term in the energy equation, we get a simple and easily solvable equation. The equation becomes:

$$
\dot{R}^{2}-H^{2} R^{2}=2 E
$$

where $H^{2}=\frac{\Lambda}{3}$ (assuming $\Lambda>0$ ) This equation can be solved in quadrature, and the solutions are deSitter-like solutions

$$
R(r, t)= \begin{cases}\frac{\sqrt{2 E(r)}}{H} \sinh \left(H\left(t-t_{0}(r)\right)\right), & E>0 \\ e^{H\left(t-t_{0}(r)\right)}, & E=0 \\ \frac{\sqrt{2|E(r)|}}{H} \cosh \left(H\left(t-t_{0}(r)\right),\right. & E<0\end{cases}
$$

Small R: Neglecting the $\Lambda$ term

If the $\Lambda$ term is neglected, we end up with a energy equation which looks like:

$$
\dot{R}^{2}-\frac{2 m}{R}=2 E
$$

Introducing a parameter $\Theta$ we can also solve this equation in quadrature:

$$
\begin{aligned}
& \left\{\begin{array}{ccc}
R & =\frac{m}{2 E}(\cosh \Theta-1) \\
t-t_{0}(r) & =\frac{m}{(2 E)^{\frac{3}{2}}}(\sinh \Theta-\Theta)
\end{array}\right\}, E>0 \\
& R=\left(\frac{9}{2} m\right)^{\frac{1}{3}}\left(t-t_{0}(r)\right)^{\frac{2}{3}}, E=0 \\
& \left\{\begin{array}{ccc}
R & =\frac{m}{2|E|}(1-\cos \Theta) \\
t-t_{0}(r) & =\frac{m}{(2|E|)^{\frac{3}{2}}}(\Theta-\sin \Theta)
\end{array}\right\}, E<0
\end{aligned}
$$

From these solutions we notice that all the small $R$ solutions goes as $R \approx\left(\frac{9}{2} m\right)^{\frac{1}{3}}\left(t-t_{0}(r)\right)^{\frac{2}{3}}$ as $R \longrightarrow 0$. In the special case $t_{0}(r)=$ constant and $F^{2}=1-k r^{2}$ the equations 32, 33 and 34 reduces to the well known matter dominated homogeneous FRW solutions.

\footnotetext{
${ }^{10}$ For a rich and qualitative description of various deSitter models see for instance [24]
} 
The classical solutions will move on level curves of the energy function $E(R, \dot{R})=\frac{1}{2} \dot{R}^{2}-$ $\frac{m}{R}-\frac{\Lambda}{6} R^{2}$, since the total energy $E$ is independent of $t$. In figure 3 the level curves of a typical energy function are drawn. If $\Lambda>0$ there will exist a saddle-point of the energy function. $\mathrm{T}$ his saddle point will be at $R=\left(\frac{3 m}{\Lambda}\right)^{\frac{1}{3}}, \dot{R}=0$ where the energy function will have the value $E_{s}=-\frac{1}{2}\left(9 m^{2} \Lambda\right)^{\frac{1}{3}}$. The saddle point solution is static, and is as a matter of fact the Einstein static universe $\square$. If $E<E_{s}$, the solutions fall into two distinct classes:

1. Schwarzschild-like solutions: These solutions expands, but they do not possess enough energy to escape the gravitational collapse, so they end as black holes. If $m$ is constant these solutions are those of a Schwarzschild black hole in Lemaitre coordinates.

2. deSitter-like solutions: solutions where the universe evolves approximately as that of deSitter solutions with positively curved hypersurfaces.

If $E>E_{s}$ the (test) matter has enough energy to escape the gravitational collapse (expanding solutions) or enough energy to prevent the gravitational repulsion from the cosmological constant (contracting solutions).

NOTE:

All of these solutions are also valid for the case $F=0$ the KS case. But to obtain the $\mathrm{KS}$ solutions it is not enough to solve just the mass equation.

\section{THE QUANTUM MECHANICAL LT MODEL}

From here on we will only consider the case where the cosmological constant is nonnegative. As we saw from the classical case, if the "energy" is small enough the solutions are confined to be either Schwarzschild-like or deSitter-like. These two regions are separated by a classically forbidden region. From classical quantum mechanics we have learned that this does not have to be the case for a quantum system. A quantum mechanical system may tunnel though classical barriers. In our case this means that a small Schwarzscild-like universe may tunnel though the potential barrier to become a deSitter-like universe! This has striking consequences. If we imagine that a "mother" universe could spontaneously create Schwarzschild-like black holes of less than a Planck-length, there will be a non-zero probability that one of these will tunnel through the potential barrier, and end up in an ever-expanding deSitter state. These considerations are of course rough and based on classical quantum mechanics. Whether this scenario is probable in the yet unknown theory of

\footnotetext{
${ }^{11}$ This is easily seen if we define the new radial variable to be $R$. This can be done since we have to assume that $m^{\prime}(r)>0$ on physical grounds. In the case $m^{\prime}(r)=0$, the metric become degenerate.
} 
Quantum Gravity remains to be proven. However we will use a simplified version of the WD equation and estimate the form of the wave function in various limits. We will from now on consider only the spherically symmetric case, i.e. $\kappa=1$.

\section{A. The canonical Dirac-quantization}

The Dirac quantization procedure is to let the constraint equations become operator equations of the wave function:

$$
\begin{aligned}
\hat{\mathfrak{H}} \Psi & =0 \\
\hat{\mathfrak{H}}_{r} \Psi & =0
\end{aligned}
$$

The first of these equations is the Wheeler-DeWitt equation. In addition we have the momentum constraint equation. These two equations are functional differential equations and thus in general very difficult to solve. KKTY managed in the case $\rho=0$ to find a couple of special cases where the wave function was exactly derived and interpreted [25]. Since the mass equation is a special combination of the momentum and energy constraint, the mass equation itself can be considered as a constraint. The wave function has to obey:

$$
\hat{M} \Psi=m \Psi
$$

The wavefunction has to be an eigenfunctional of the mass-operator $\hat{M}$ with eigenvalue $m(r)$, and from the classical interpretation we have to assume that the eigenvalue $m(r)$ has to obey $m^{\prime}(r) \geq 0$.

In the WKB approximation we can integrate the Hamilton-Jacobi equation if we choose a particular and simple path [26]. We will not use these results. Neither will we give any attempt to solve all three constraint equations simultaneously in the "correct" quantummechanical manner. To simplify the equations we will do the following:

- Solve the momentum constraint classically. When we solve it we will assume that $R^{\prime} \neq 0$, thus the KS case is excluded. Solving the momentum constraint will reduce the number of canonical variables, so we end up with only $R$ and its canonical conjugated momentum $\Pi_{R}$.

- Choose the gauge $N^{\prime}=0$ so that we will not have any problems with the function $F$ being dependent of $t$.

- Find the new Hamiltonian which reproduces the classical equations.

The reduced action which reproduces the classical equations is:

$$
S=\int d t \int d r \frac{N}{F}\left[-\frac{1}{2} \frac{R \dot{R}^{2}}{N^{2}}+\frac{1}{2} R\left(1-F^{2}\right)-\frac{\Lambda}{6} R^{3}-m(r)\right]^{\prime}
$$

Calculating the canonical momentum

$$
\Pi_{R} \equiv \frac{\delta S}{\delta \dot{R}}=\left(\frac{N}{F}\right)^{\prime} \frac{R \dot{R}}{N^{2}}
$$


doing a Legrendre transform to bring the action onto a canonical form, and removing boundary terms, we arrive at the Hamiltonian which reproduces the classical equations:

$$
H=\int d r \frac{N}{F}\left[-\frac{1}{2} \frac{F^{4} \Pi_{R}^{2}}{R F^{2}}-\frac{1}{2} R\left(1-F^{2}\right)+\frac{\Lambda}{6} R^{3}+m(r)\right]^{\prime}
$$

The constraint equation will now yield the correct classical equation; the mass equation. Calculating the equation for $\Pi_{R}$, we find the identity, $\dot{M}=0$, which tells us that the mass function is independent of time.

The wave function has to satisfy the constraint equation:

$$
\frac{\partial}{\partial r} \hat{\mathfrak{H}} \Psi=0
$$

where

$$
\hat{\mathfrak{H}}=\frac{1}{2} \frac{F^{4}}{R F^{\prime 2}} \frac{\delta^{2}}{\delta R^{2}}-\frac{1}{2} R\left(1-F^{2}\right)+\frac{\Lambda}{6} R^{3}+m(r)
$$

A possible constant, due to the derivative with respect to $r$ is assumed to be absorbed into the function $m$.

This simplified version of the LT model is not only simpler but the action has the great advantage that it reduces to the deSitter models studied by Vilenkin and Hartle-Hawking if we demand that $m=0, R=a(t) r$ and $F^{2}=1-k r^{2}$. The solution most likely to describe the tunneling behaviour emphasized in the beginning of this section, is the tunneling wavefunction suggested by Vilenkin.

\section{B. The tunneling wave function in the WKB approximation}

In the WKB approximation we assume that the wave function has the form $\Psi_{W K B}=$ $\exp ( \pm i S)$, to the lowest order we get the Hamilton-Jacobi equation:

$$
\left(\frac{\delta S}{\delta R}\right)^{2}-\frac{F^{\prime 2}}{F^{4}}\left[2 m R-R^{2}\left(1-F^{2}\right)+\frac{\Lambda}{3} R^{4}\right]=0
$$

If we assume that $S=\int \sigma(r) d r$ the resulting equation will be the Hamilton-Jacobi equation for a point particle with action $\sigma(r)$ ( $r$ is only a parameter). In the Hamilton-Jacobi equation the functional $S$ turn out to be the action at the classical level. Since the classical action can be written as an integral over $r$ the assumption $S=\int \sigma(r) d r$ is therefore reasonable at the lowest order WKB level. This separation of the action is also a feature of the dust, in the sense that dust does not self-interact. Because the matter does not self-interact the matter

\footnotetext{
${ }^{12}$ Inserting $m=\frac{4}{3} \pi \hat{\rho} r^{3}, R=a(t) r$ and $F^{2}=1-k r^{2}$ where $\hat{\rho}$ and $k$ are constants into the action, the $r$ dependent part can be factored out, and the integral may be performed. The actual integral obtained is: $\int d r \frac{r^{2}}{\sqrt{1-k r^{2}}}=\frac{\pi}{4 k^{\frac{3}{2}}}$ in the case of $k>0$.
} 
equations do not depend on neighbouring points. We can interpret the action $\sigma$ as the action of a point particle moving in a potential $V(R)=\frac{F^{2}}{F^{4}}\left[-m R+\frac{1}{2}\left(1-F^{2}\right) R^{2}-\frac{\Lambda}{6} R^{4}\right]$ with zero energy. The WKB wavefunction $\psi_{W K B}$ for the point particle can then be written $\psi_{W K B}=\exp ( \pm i \sigma)$. The two WKB wavefunctions can therefore be related by $\Psi_{W K B}=\exp \left(\int d r \ln \psi_{W K B}\right)$. Finding first the wavefunction $\psi$ we can then relate its WKB approximation with $\Psi_{W K B}$ through $\Psi_{W K B}=\exp \left(\int d r \ln \psi_{W K B}\right)$.

We imagine that the universe point starts off at $R=0$ and the behavior of the wave function of such a particle is dependent on the form of the potential. The zeros of the potential indicate classical turning points, separating classically allowed/forbidden regions. The potential $V(R)$ has one zero at $R=0$ and possibly two more for positive values of $R$. Whether there are two, one or zero, depends on the entity:

$$
l=\frac{9 m^{2} \Lambda}{\left(1-F^{2}\right)^{3}}
$$

Iff $0<l<1$ then there will be two positive roots, $r_{1}$ and $r_{2}$. The region $r_{1}<R<r_{2}$ (assuming $r_{1}<r_{2}$ ) is a classical forbidden region, thus the wavefunction is exponential in some way. Iff $l=1$ these two roots have emerged into one, and the case $l>1$ and $l<0$ has no positive roots; the classical particle rolls down the slope to infinity.

One case which is very instructive to look at is the case where $F^{2}=1-k r^{2}$ and the dust density is constant: $m=\frac{4}{3} \pi \hat{\rho} r^{3}$. The entity $l$ will then be a constant with respect to $r$ :

$$
l=\frac{16 \pi^{2} \hat{\rho}^{2} \Lambda}{k^{3}}
$$

We can interpret this quantum mechanically as if we imagine a spontaneously created universe "bubble" with high curvature, and a relatively small cosmological constant and dust density. It is most likely to vanish before it has the opportunity to enter a deSitter phase. Let us look at this from another point of view. Imagine that we can only observe "big" universes, universes at scales much larger than the Planck length, or essentially just universes that are in the expanding deSitter stage. If we could in some way sit outside and see the flow of universes in expanding deSitter stages, we would find that most of the universes with low $\Lambda$ and $\hat{\rho}$ also have low hypersurface curvature.

Let these thought experiments be for the moment because we do not actually know how to interpret the wave function of the universe or how to calculate it. Let us however try to estimate the behavior of the wavefunction is this model.

The most interesting case seems to be $0<l<1$, so let us just look at this case. There are essentially three different regions with different behavior.

1. Region I: Small $R$. We can neglect the $R^{4}$-term.

2. Region II: Intermediate region. This is dominated by the part where the wave function has an exponential behavior.

3. Region III: Large $R$. We can neglect the $R$-term. 


\section{Region I}

We neglect the $R^{4}$-term in eq. 40 and complete the square with a new variable $\frac{F}{\sqrt{\left|F^{\prime}\right|}} \lambda=$ $\left(1-F^{2}\right)^{\frac{1}{4}} R-\frac{m}{\left(1-F^{2}\right)^{\frac{3}{4}}}$. Then the Schrödinger equation for $\psi$ reduces to a Harmonic oscillatorlike equation:

$$
\frac{\partial^{2} \psi}{\partial \lambda^{2}}+\left(2 p+1-\lambda^{2}\right) \psi=0
$$

where $p=\frac{1}{2}\left(\frac{\left|F^{\prime}\right| m^{2}}{F^{2}\left(1-F^{2}\right)^{\frac{3}{2}}}-1\right)$. The solution of this equation can be written in terms of the Parabolic cylinder functions $D_{p}(z)$ :

$$
\psi=\left\{\begin{array}{l}
D_{p}( \pm \sqrt{2} \lambda) \\
D_{-p-1}( \pm i \sqrt{2} \lambda)
\end{array}\right.
$$

In the case of $p=n$ an integer, the $D_{p}(z)$ can be written in terms of the Hermite polynomials (as one would expect):

$$
D_{n}(z)=-2^{-\frac{n}{2}} e^{-\frac{z^{2}}{4}} H_{n}\left(\frac{z}{\sqrt{2}}\right)
$$

The index $p$ can be any number, in contrast to the 1-dimensional Harmonic oscillator.

\section{Region III}

We neglect the $R$-term in eq. 40. This is equivalent to setting the mass function $m$ equal to zero. We are then left with a deSitter space-time. The wavefunction for such space-times has been calculated using the WKB approximation. Most interesting to us is the tunneling wave function of Vilenkin. Through the rescaling: $\eta=\frac{\sqrt{\left|F^{\prime}\right|}}{F}\left(1-F^{2}\right)^{\frac{1}{4}} R$ we can bring the equation to the same form as that of Vilenkin [27], with a scalar field potential $V=\frac{F^{2} \Lambda}{3\left|F^{\prime}\right|\left(1-F^{2}\right)^{\frac{3}{2}}}$. We use this result to obtain:

$$
\psi \approx \begin{cases}\exp \left(-\frac{1}{3 V}\left[1-\left(1-\frac{R^{2} \Lambda}{3\left(1-F^{2}\right)}\right)^{\frac{3}{2}}\right]\right), & \frac{R^{2} \Lambda}{3\left(1-F^{2}\right)}<1 \\ \exp \left(-\frac{1}{3 V}\left[1+i\left(\frac{R^{2} \Lambda}{3\left(1-F^{2}\right)}-1\right)^{\frac{3}{2}}\right]\right), & \frac{R^{2} \Lambda}{3\left(1-F^{2}\right)}>1\end{cases}
$$

Let us assume that $m(r)=0$ and $F^{2}=1-k r^{2}$ where $k$ is assumed to be positive. We will now use the tunneling solution eq. 41 to find out what is most likely, the creation of a highly curved or a less curved space. If we calculate the ratio $\frac{\left.\Psi^{*} \Psi\right|_{R=\tilde{R}}}{\left.\Psi^{*} \Psi\right|_{R=0}}$ where $\tilde{R}^{2}>>\frac{3}{\Lambda}\left(1-F^{2}\right)$, we get:

$$
P_{k} \equiv \frac{\left.\Psi^{*} \Psi\right|_{R=\tilde{R}}}{\left.\Psi^{*} \Psi\right|_{R=0}}=\exp \left[-\frac{2}{\Lambda} \int_{0}^{1} d(\sin \theta) G(\theta)\right]
$$


where $G$ is a real function independent of $k$ which satisfies $G(\theta) \geq 0, \forall \theta$. We thus have to conclude that to lowest order in the WKB approximation it is equally probable for a space with small spatial curvature to tunnel through the classical barrier than for a highly curved space. Comparing with our previous statement, we se that larger curvarture itself increases the probability amplitude. But smaller curvature increases the volume by exactly the amount so that it cancels the contribution from the curvature.

\section{Region II}

Here the wave function will be of exponential behavior. But the actual shape is strongly dependent on the boundary condition. To find the wave function in this region to lowest order, the best is perhaps to use the function from Region I and III and cut and glue them together.

\section{Connection with other work}

Nordbury 28,29 studied the FRW model with different types of perfect fluids. The tunneling picture which we have described here seems to agree with his results for a closed universe $\left(1-F^{2}>0\right)$. However in the case of open $(k=-1)$ FRW-universes he concludes that these models can not have been created through the tunneling picture. From our considerations however, this can not be ruled out, on the contrary, open universes do seem to tunnel from a $R=0$ state a lot easier than closed ones.

Also an article by Atkatz and Pagels [6] seems to indicate that open universes can not be created by a tunneling event. A key argument used in both of these articles is that the transition amplitude is suppressed by the infinite integral over the three-dimensional hypersurfaces. However, a more recent article by Coule and Martin [30] used the fact that there exists $k=-1$ compact spacelike hypersurfaces. Since they are compact they will have finite volume. Their conclusion is opposite of that of the previous two articles: The creation of a open $k=-1$ FRW universe is more likely than a closed $k=1$ universe through a tunneling event. Our case is however more subtle, since the hypersurfaces are no longer homogeneous.

\section{Inhomogeneity Vs. Homogeneity: Tunneling Probabilities}

As we have investigated the WKB wavefunctions in this model, we might wonder whether an inhomogeneous universe is more likely to be created through a tunneling event than a homogeneous one. As an inhomogeneity measure in this model it is very useful to use the Weyl curvature tensor $C_{\alpha \beta \gamma \delta}$. All of the FRW models are conformally flat, thus for the all

of the FRW model the Weyl tensor will vanish. In our case the opposite is also true, if $C_{\alpha \beta \gamma \delta}=0$ and $N^{\prime}=0$ in the LT model then the spacetime is spatially homogeneous. If we define a mean dust density function $\bar{\rho}$ by:

$$
m(r)=\frac{4}{3} \pi \bar{\rho} R^{3}
$$


we can write the Weyl curvature invariant in the LT model as:

$$
C^{\alpha \beta \gamma \delta} C_{\alpha \beta \gamma \delta}=\frac{16^{2}}{3} \pi^{2}(\bar{\rho}-\rho)^{2}
$$

Thus this space-time is homogeneous if and only if $\bar{\rho} \equiv \rho$.

The inhomogeneity in this model is therefore almost completely characterized by the mass function $m(r)$. The homogeneous mass function will go as $m_{h}(r)=K r^{3}$, where $K$ is a constant. Investigating only closed universes, we fix the constant $K$ so that $m_{h}\left(r_{\max }\right)=$ $m\left(r_{\max }\right)$. The most physical interesting universes that these LT models describe will have a larger amount of dust consentrated in the inner regions than the outher regions. The most extreme inhomogeneous mass function is the Schwarzschild mass function, $m_{S c h}(r)=\mu=$ constant. Thus it is very reasonable to assume that we have a mass distribution that obeys:

$$
m_{S c h}(r) \geq m(r) \geq m_{h}(r)
$$

If we compare the WKB tunneling potential $V(R)=\frac{F^{\prime 2}}{F^{4}}\left[-m R+\frac{1}{2}\left(1-F^{2}\right) R^{2}-\frac{\Lambda}{6} R^{4}\right]$ for these different mass distributions we see that we get

$$
V_{S c h}(R) \leq V(R) \leq V_{h}(R)
$$

keeping all other parameters fixed. Thus the more inhomogeneous the space is the smaller the tunneling barrier will be. In the WKB approximation this means that the probability of the creation of an inhomogeneous universe is larger than for a homogeneous universe. More specific the spontaneous creation of a Schwarzschild-deSitter like universe appears to be more probable than a homogeneous FRW universe. We also have be aware of that the matter Lagrangian describes dust. Dust is an idealised form of matter that has no internal pressure. As the dust density becomes high, either in an early stage of the universe or in large local inhomogeneities this assumption of a pressure-free matter Langrangian becomes somewhat artificial and unphysical. In these situations we know that the internal pressure becomes very important and even quantum effects from the matter fields are essential in the description. In this sence we may say that these calculations more or less are "valid" only with small local inhomogeneities or they describe the effect from Gravity itself to produce inhomogeneities. This point is also emphasized by Conradi [31,32. Conradi discusses the wave function of an anisotropic KS space with a dust Lagrangian. He argues that since dust interacts only locally, in the sence that it does not self-interact, this local "tunneling" process cannot be interpreted as a tunneling effect. Our results should therefore perhaps be seen from a more phenomological point of view. The matter Lagrangian used in this paper is one of the most simple source field that give rise to inhomogeneities. The source field used is also interesting because it connects the Schwarzschild spacetime with the FRW spacetime in a smooth way.

\section{CONCLUSION AND SUMMARY}

In a Hamiltonian description of General Relativity there has been a consensus among physicists and cosmologists that the issue of topology is essential and necessary in the description [33,34]. This has also been emphasized in this paper. At the classical level we saw 
that apparently different cases would lead to the same spacetime if the symmetry spaces were infinite and simply connected. Assuming compactness and multiply connectedness this pathology was removed and yielded some interesting spacetimes.

In the Quantum mechanical case we provided a simpler model which was in general inhomogeneous, but removed many of the algebraic difficulties in the full KSLT model. It was emphasized how this model could describe a quantum creation of a inhomogeneous universe through quantum tunneling. We also showed that under some reasonable assumptions the creation of an inhomogeneous universe was more probable than a homogeneous FRW universe. In the absence of interacting matter this is very reasonable because there are a lot more inhomogeneous configurations than homogeneous ones.

Even though the full theory of Quantum Gravity is far from being established, it seems like Quantum Cosmology could describe many of the features of the initial universe. In the recent decade the interest for topology in cosmology has expanded the application for

Quantum Cosmology. Even in classical gravity the inclusion of non-trivial topologies has yielded new and surprising results.

\section{ACKNOWLEDGMENTS}

I deeply appreciate the help and comments which Øyvind Grøn has given me during the work of this paper.

I also thank Branko Steffensen for reading through the manuscript and making useful comments. 


\section{REFERENCES}

[1] A. Vilenkin, Phys. Lett. B117, 25 (1982)

[2] A. Vilenkin, Phys. Rev. D27, 2848 (1983)

[3] A. Vilenkin, Phys. Rev. D30, 509 (1984)

[4] A. Vilenkin, Phys. Rev. D33, 3560 (1986)

[5] T. Vachaspati and A. Vilenkin, Phys. Rev. D37, 898 (1988)

[6] D. Atkatz and H. Pagels, Phys. Rev., D25, 2065 (1982)

[7] M. Lachièze-Rey and J-P. Luminet, Phys. Rep. 254, 135 (1995)

[8] W.P. Thurston, Three-Dimensional Geometry and Topology, Volume 1, Princeton University Press (1997)

[9] K. Kuchař, Phys. Rev. D50, 3961 (1994)

[10] M. Kenmoku, H. Kubotani, E. Takasugi, Y. Yamazaki, Phys. Rev. D59, (1999)

[11] W.P. Thurston, Bull. Am. Math. Soc. 6, 357 (1982)

[12] H. Fagundes, Lett. Math. Phys. 6, 417 (1982)

[13] Y. Nambu and M. Sasaki, Prog. Theo. Phys. 79, 96 (1988)

[14] K. Kuchař and C.G. Torre, Phys. Rev. D43, 419 (1991)

[15] D.S. Salopek, Phys. Rev. D46, 4373 (1992)

[16] S. Hervik, Cand. Scient. Thesis, University of Oslo (1999)

[17] D. Brill, J. Louko and P. Peldán, Phys. Rev. D56, 3600 (1997)

[18] M. Bañados, C. Teitelboim and J. Zanelli, Phys. Rev. Lett. 69 (1992)

[19] D.D. Sokoloff and A.A. Starobinskii, Sov. Astron. 19, 629 (1975)

[20] S. Hervik, Class. Quantum Grav. 17, 2765 (2000)

[21] I. Bengtsson and S. Holst, Class. Quantum Grav. 16, 3735 (1999)

[22] A. Krasiński, Inhomogeneous Cosmological models, Cambridge University Press (1997)

[23] A. Zecca, Il nuovo Cimento, 106B, 413 (1990)

[24] E. Eriksen and Ø. Grøn, Int. J. Mod. Phys. 4, 115 (1995)

[25] M. Kenmoku, H. Kubotani, E. Takasugi, Y. Yamazaki, gr-qc/9906056 (1999)

[26] W. Fischler, D. Morgan and J. Polchinski, Phys. Rev. D42, 4042 (1990)

[27] A. Vilenkin, Phys. Rev. D37, 888 (1988)

[28] J.W. Norbury, Eur. J. Phys. B433, 263 (1998)

[29] J.W. Norbury, Phys. Lett. 19, 143 (1997)

[30] D.H. Coule and J. Martin, Phys. Rev. D61, 063501 (2000)

[31] H-D. Conradi, Class. Quantum Grav. 12, 2423 (1995)

[32] H-D. Conradi, Int. J. Mod. Phys. D7, 189 (1998)

[33] A. Ashtekar and J. Samuel, Class. Quantum Grav. 8, 2191 (1991)

[34] H. Kodama, Prog. Theo. Phys. 99, 173 (1998) 


\section{TABLES}

\begin{tabular}{|c||c|c|c|}
\hline$\Lambda$ & $\kappa=1$ & $\kappa=0$ & $\kappa=-1$ \\
\hline \hline+ & KS-S-dS-KS & KS & KS \\
\hline 0 & KS-Sch & KS & KS \\
\hline- & KS-AdS & KS-AdS & KS-AdS \\
\hline
\end{tabular}

TABLE I. Summary of the classical solutions. The ones to the left are the interior solutions, to the right are exterior solutions. We also use the term KS in all cases where the spatial topology can be written as $S^{1} \times T_{g}$. Other abbreviations: S-dS: The Schwarzschild-deSitter family, Sch: The Schwarzschild solution, AdS: Anti-deSitter (or quotients of). 


\section{FIGURES}

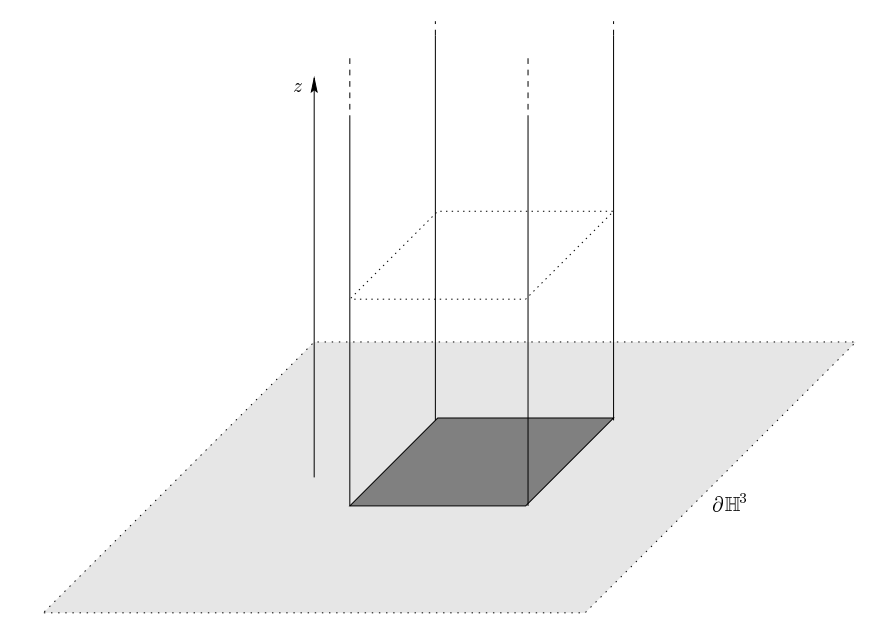

FIG. 1. The space $d s^{2}=\frac{1}{z^{2}}\left(d z^{2}+d \Omega_{1}^{2}\right)$

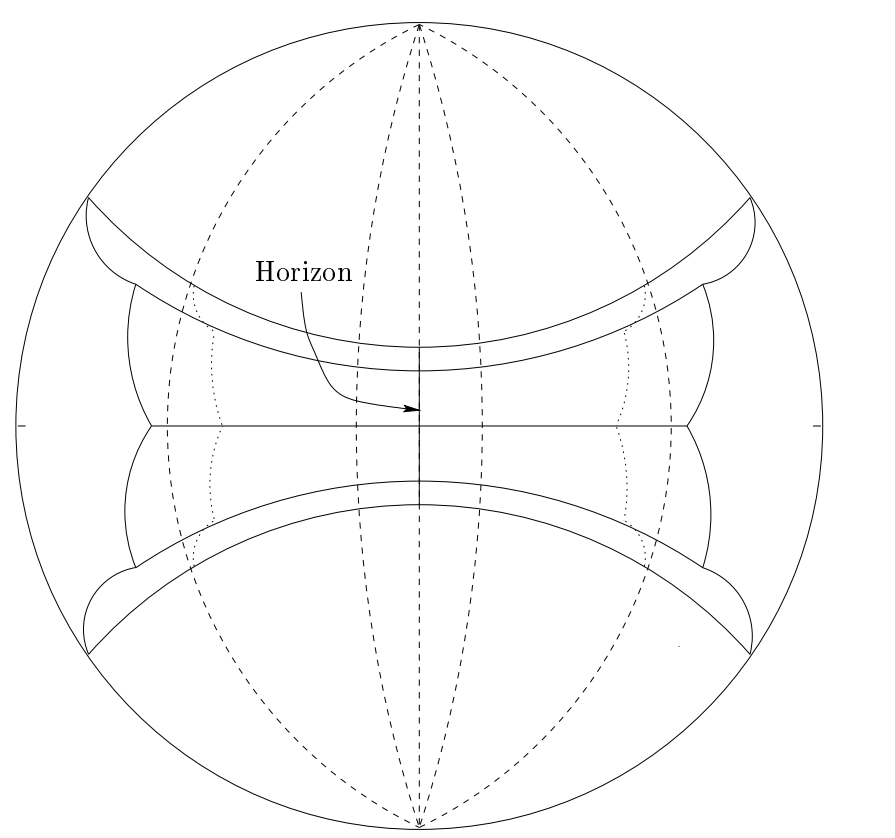

FIG. 2. The $\mathrm{AdS}_{4}$ apple. This figure is not drawn to scale. 


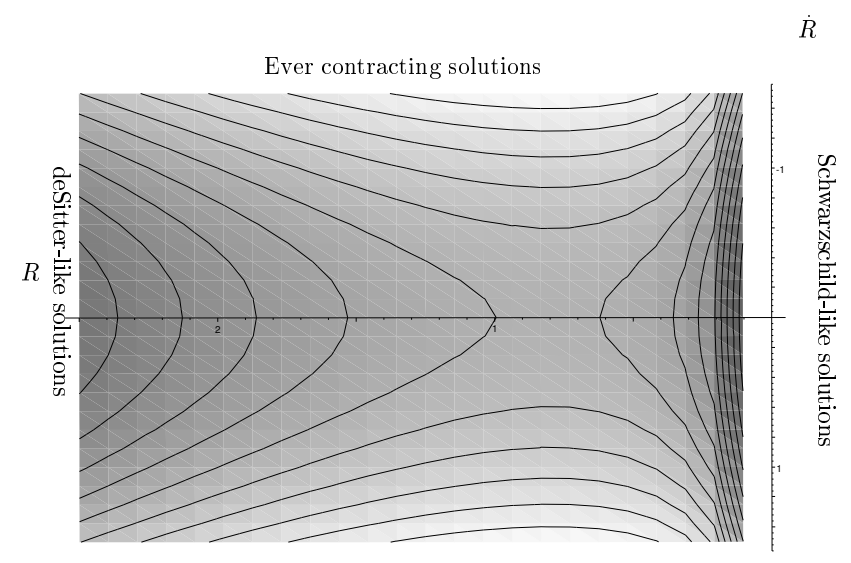

Ever expanding solutions

FIG. 3. Level curves of $E(R, \dot{R})=\frac{1}{2} \dot{R}^{2}-\frac{1}{5 R}-\frac{1}{5} R^{2}$

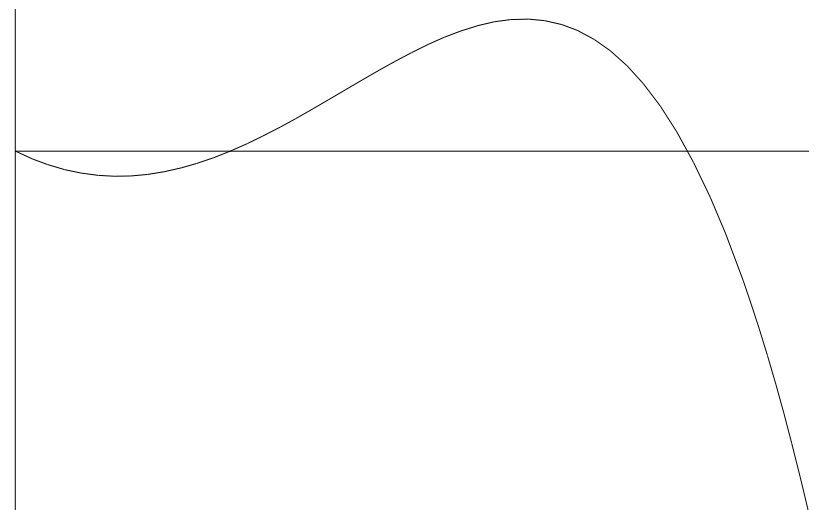

FIG. 4. The tunneling potential for the reduced LT model

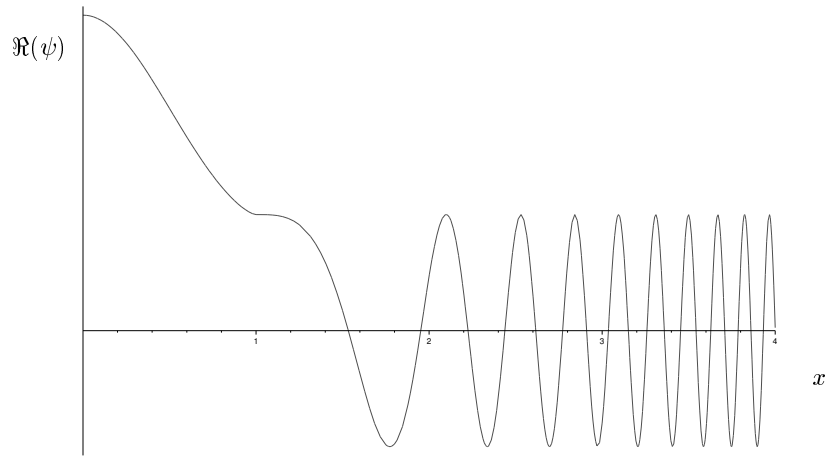

FIG. 5. The real part of the tunneling wavefunction of Vilenkin eq. 41 